\title{
Love Wave Device for Real-Time Monitoring of Pollutant Degradation through Photocatalysis
}

\author{
L. Blanc ${ }^{1}$, A. Tetelin ${ }^{1}$, C. Boissière ${ }^{2}$, C. Dejous ${ }^{1}$, D. Rebière ${ }^{1}$ \\ ${ }^{1}$ Laboratoire IMS, 351 Cours de la Libération, 33400 Talence, France \\ ${ }^{2}$ LCMCP, 11 Place Marcelin-Berthelot, 75231 Paris Cedex 05, France \\ laurianne.blanc@ims-bordeaux.fr
}

\begin{abstract}
:
A mesoporous titania-coated Love wave sensor is used to monitor the real-time degradation of stearic acid through a photocatalytic reaction. The pollutant is deposited directly on the porous film. It leads, at first, to a decrease of the resonant frequency. Then, the device is placed under UV light and its frequency response is real-time monitored. After 12 hours under exposure, the frequency successfully reached its initial value.
\end{abstract}

Acoustic sensors present many advantages compared to conventional measurement systems for photocatalytic activity: real-time response, small size, and low cost. The Love wave devices could allow to monitoring the degradation kinetics of other pollutants. They could also be a useful tool to study the influence of the UV wavelength on the degradation rate.

Key words: Photocatalytic reaction, Love wave sensor, real-time pollutant degradation.

\section{Introduction}

Conventional tests for the measurement of photocatalytic activity require bulky and expensive equipment. Nonetheless, Nakamura et al. [1] have shown that it was possible to follow the real-time degradation of a pollutant with a Quartz Crystal Microbalance (QCM) coated with a titania layer. In particular, when this layer is cracked, the sensitivity of the sensor is improved.

In this work, the aim is to show the ability of surface acoustic wave sensors to measure the degradation of a pollutant in real time. In particular, Love wave sensors present a better sensitivity than QCM due to their shear horizontal wave propagation in a guiding layer. Moreover, acoustic sensors present many advantages compared to conventional measurement systems for photocatalytic activity: real-time response, small size, and low cost.

Love wave devices are widely used for chemical detection in gaseous and liquid media. Recently, Love wave devices coated with mesoporous layers have presented high sensitivities for volatile organic compound detection [2] and for heavy metal detection [3].

$\mathrm{TiO}_{2}$ is also largely used as a photocatalyst due to its high electron mobility [4]. This semiconductor material enables to decompose pollutants exposed to ultraviolet radiations, when the wavelength $(\lambda)$ of UV is greater than the $\mathrm{TiO}_{2}$ bandgap (hv), with $h v>3,2 \mathrm{eV}$ or $\lambda<380 \mathrm{~nm}$. Moreover, the photocatalytic activity of $\mathrm{TiO}_{2}$ strongly depends on its surface area, its crystalline structure and its synthesis method [4]. Mesoporous titania offers a large number of active sites where catalytic reactions may take place. Note that the mesoporous layer is synthesized with the evaporation-induced self-assembly (EISA) process [5].

After a short description of the Love wave platform, the characteristics of the titania mesoporous film, determined through porosimetric ellipsometry, are presented. Then, the PDMS cell, used for the deposition of pollutants on the sensor, is presented [6]. Finally, the experimental results are displayed and interpreted.

\section{Love wave platform}

The Love wave sensor was fabricated on a $500 \mu \mathrm{m}$ AT cut Quartz substrate. The Ti/Au interdigitated transducers (IDTs), generating the shear horizontal surface acoustic waves, consisted of 44 finger pairs with a $40 \mu \mathrm{m}$ periodicity $(\lambda)$ and an acoustic path (between both transducers) of $164 \lambda$. In order to generate Love waves and to confine acoustic energy, a $4.5 \mu \mathrm{m} \mathrm{SiO}{ }_{2}$ guiding layer was deposited on the top of the Quartz substrate. The addition of a 
sensitive layer on the propagation path enabled the specific sorption of molecules. The sorption modifies the physico-chemical properties of the layer, which are measured through the variations of the phase velocity.

For high resolution experimental measurements, the Love wave delay-line is inserted in the feedback loop of a radiofrequency amplifier to achieve an oscillator system, and the measured frequency shifts are linked to the wave velocity shifts. Note that each sensor was composed of two delay-lines.

\section{$\mathrm{TiO}_{2}$ mesoporous layer}

The titania mesoporous film was prepared via the EISA sol-gel-based process, which enables the full control of the film structural properties [5]. The precursor solution contained $20.66 \mathrm{~g}$ of ethanol, $1.45 \mathrm{~g}$ of acid water ( $\mathrm{pH} 2 \mathrm{M} / 36), 0.56 \mathrm{~g}$ of $\mathrm{F} 127$ surfactant and $3.347 \mathrm{~g}$ of a solution containing a ratio of one $\mathrm{TiCl} 4$ molecule to five EtOH molecules.

The solution was spread by spin-coating on the acoustic path of the sensor (Fig. 1). Then, the device spent 10 hours in a climatic chamber set at $75 \% \mathrm{RH}$ (relative humidity) at ambient temperature. A dedicated thermal cure is finally applied to obtain a homogeneous porous film: first, from ambient to $400{ }^{\circ} \mathrm{C}$ with a speed of $5{ }^{\circ} \mathrm{C} / \mathrm{min}$; then, five hours at $400{ }^{\circ} \mathrm{C}$; and finally, back to the ambient temperature at the same rate.

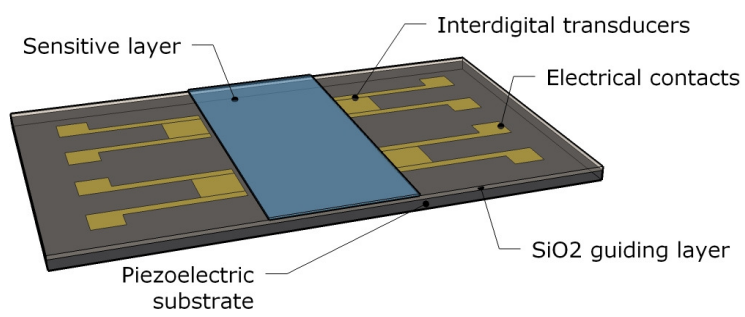

Fig.1: Scheme of a Love wave platform including two delay-line

Eventually, the mesoporous layer has been characterized through environmental ellipsometric porosimetry [7], which showed a porosity of $25 \%$ with an average pore size of $9.8 \mathrm{~nm} \times 12 \mathrm{~nm}$ providing a large contact surface area with the pollutant (Fig. 2). The film thickness was about $110 \mathrm{~nm}$.

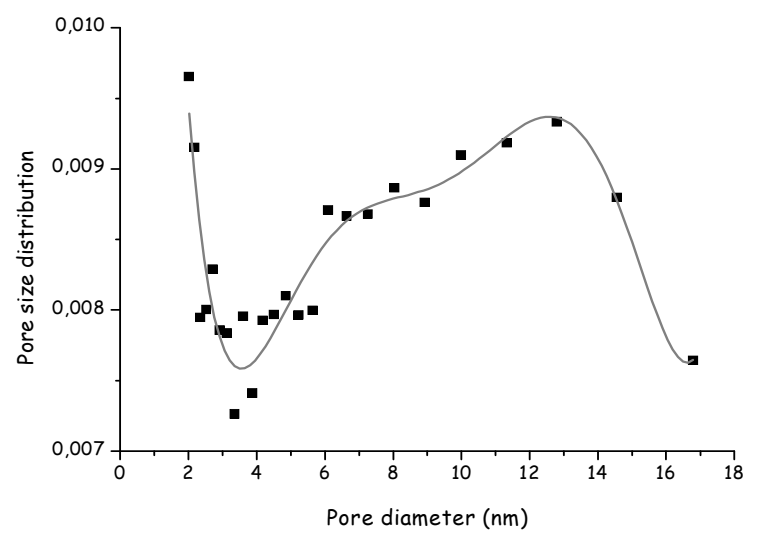

Fig.2: Pore size distribution of a $110 \mathrm{~nm} \mathrm{TiO}_{2}$ layer on a Love wave device measured through EEP@18 ${ }^{\circ}$

\section{PDMS cell}

Poly (dimethylsiloxane) (PDMS) polymer is widely used for sensor applications because of its biocompatibility, ease of cleaning, and reusability $[8,9]$. The considered PDMS cell (Fig. 3) has been designed to delimit a specific coated zone for pollutant deposition on the acoustic path [6]. It also enables to isolate the IDTs from the liquid through a protective air cavity. Moreover, the cell is maintained by pressure on the sensor, which allows easy cleaning and to reuse the acoustic platform.

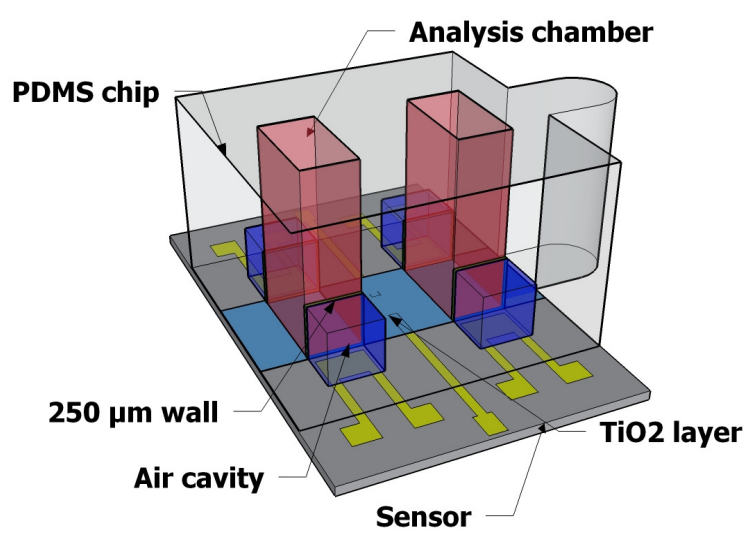

Fig.3: PDMS cell with analysis chambers fixed on the sensor

\section{Real-time monitoring of stearic acid degradation}

The stearic acid was mixed in 10:1 weight ratio (ethanol : $\mathrm{C}_{18} \mathrm{OH}$ ). $2 \mu \mathrm{L}$ was injected on the $\mathrm{TiO}_{2}$ film through the analysis chamber with a micropipette. Then, the solvent contained in the solution evaporates leading to the saturation of the $\mathrm{TiO}_{2}$ film with stearic acid. The device was placed under $365 \mathrm{~nm}$ wavelength UV light for $16 \mathrm{~h}$.

The degradation of the pollutant results in a mass change on the Love wave sensor and is real-time monitored through the frequency 
response of the device, whose resonant frequency is close to $118 \mathrm{MHz}$.

Fig. $2 a$ shows the frequency shifts induced by the deposition and the subsequent degradation of the stearic acid $\left(\mathrm{C}_{18} \mathrm{OH}\right)$. At first, a frequency decrease of $140 \mathrm{kHz}$ is induced by the pollutant. When exposed to UV, the resonant frequency increases until it reaches its initial value back, thus showing the total degradation of the pollutant after $12 \mathrm{~h}$ of UV exposure. The first and reproducible frequency shift (Fig. $2 b$ ) is due to the evaporation of the solvents contained in the solution of stearic acid.

When the device is not exposed to UV light, the frequency increases as the solvent evaporates, and stabilizes after $2 \mathrm{~min}$. This reference test suggests that the recorded frequency variations should successfully follow the decomposition of the pollutant.

a)

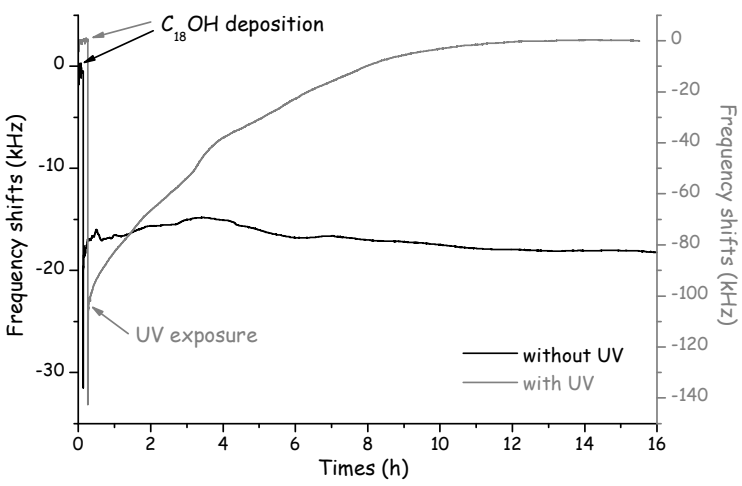

b)

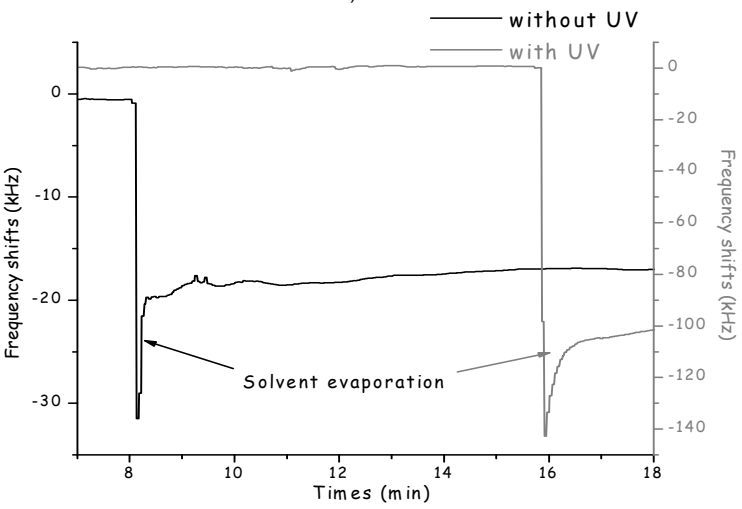

Fig.2: a) Evolution of the resonant frequency of a Love wave device coated with a $\mathrm{TiO}_{2}$ layer after deposition of the pollutant and exposed (or not) to UV light (wavelength: $365 \mathrm{~nm}$ )

b) Zoom on the evaporation of the solvent contained in the stearic acid solution

\section{Conclusion}

This work has presented the feasibility of using a Love wave sensor to monitor the degradation of a pollutant by photocatalytic reactions in realtime.

The sensor was coated with mesoporous $\mathrm{TiO}_{2}$, because this material is widely used as a photocatalyst due to its physical, chemical and optical properties. Moreover, used in its mesoporous form, it offers a large contact surface area with the pollutant.

Experimental results have shown a frequency shift of $140 \mathrm{kHz}$ after stearic acid deposition, After $12 \mathrm{~h}$ under $365 \mathrm{~nm}$ wavelength UV exposure, the resonant frequency returns to its initial value, which corresponds to the full degradation of the pollutant.

\section{References}

[1] Y. Nakamura, Y. Katou, S. Rengakuji, Electrochemistry, 72, 408-11 (2004)

[2] G. Tortissier, L. Blanc, A. Tetelin, C. Zimmermann, J.-L. Lachaud, C. Boissière, C. Sanchez, C. Dejous, D. Rebière, Sensors Letters, 7, 1-5 (2009); doi: 10.1166/sl.2009.1185

[3] L. Blanc, I. Gammoudi, H. Tarbague, C. Boissière, R. Kalfat, C. Dejous, A. Tetelin, D. Rebière, ECS Transactions, 39, 337-343 (2011); doi : 10.1149/1.3615211

[4] X.X. Fan, T. Yu, L.Z. Zhang, X.Y. Chen, Z.G. Zou, Chinese Journal of Chemical Physics, 20, 6, 733-38 (2007); doi:10.1088/16740068/20/06/733-738

[5] D. Grosso, F. Cagnol, G.J. de A.A. Soler-Illia, E.L. Crepaldi, H. Amenitsch, A. Brunet-Bruneau, A. Bourgeois, C. Sanchez, Advanced Functional Materials, 14, 309-322 (2004); doi: 10.1002/adfm 200305036

[6] H. Tarbague, J.-L. Lachaud, S. Destor, L. Vellutini, J.-P. Pillot, B. Bennetau, E. Pascal, D. Moynet, D. Mossalayi, D. Rebière, C. Dejous, Journal Integrated Circuits and Systems, 5, 125133 (2010);

[7] C. Boissière, D. Grosso, S. Lepoutre, L. Nicole, A. Brunet Bruneau, C. Sanchez, Langmuir, 21, 12362-12371 (2005); doi: 10.1021/la050981z

[8] B. T. Ginn, B. Steinbock, M. Kahveci, O. Steinbock, Journal of Physical Chemistry A, 108, 1325-1332 (2004); doi: 10.1021/jp0358883

[9] N. Kim, C. M. Dempsey, J. V. Zoval, J.-Y. Sze, M. J. Madou, Sensors and Actuators B, 122, 511518 (2007); doi: 10.1016/j.snb.2006.06.026 